\title{
A simple technique for reconstruction of medial patellofemoral ligament with bone-fascia tunnel fixation at the medial margin of the patella: a 6-year-minimum follow-up study
}

Jia $\mathrm{Li}^{1 \dagger}$, Yongqian $\mathrm{Li}^{1+}$, Jingchao Wei ${ }^{2}$, Jianzhao Wang ${ }^{1}$, Shijun Gao ${ }^{1}$ and Yong Shen ${ }^{1 *}$

\begin{abstract}
Background: Medial patellofemoral ligament (MPFL) reconstruction has become an accepted technique to treat patellofemoral instability, and numerous surgical techniques have been described to reconstruct the MPFL. We describe a MPFL reconstruction procedure where bone-fascia tunnel fixation occurs at the medial margin of the patella for recurrent patellar dislocation.
\end{abstract}

Objective: MPFL reconstruction is the preferred operative treatment for recurrent patellar dislocation. The purpose of this study was to report a simple technique for reconstruction of medial patellofemoral ligament with bone-fascia tunnel fixation at the medial margin of the patella for recurrent patellar dislocation and to evaluate the results at 6-year-minimum follow-up.

Methods: The study included 65 patients (28 males, 37 females; mean age, $29.4 \pm 5.6$ years) who underwent MPFL reconstruction using the bone-fascia tunnel fixation at the medial margin of the patella technique and who were followed for a mean duration of $78.5 \pm 3.8$ months. Objective assessment, Kujala scale, Lysholm score, and Tegner activity score were obtained preoperatively and the time of final follow-up.

Results: There were no patellar complications, including redislocation, in the present study. The congruence angle had significant improvement from $19.2^{\circ} \pm 6.3^{\circ}$ before surgery to $-6.03^{\circ} \pm 0.50^{\circ}$ at the last follow-up. The lateral patellar angle had significant improvement from $-6.9^{\circ} \pm 3.5^{\circ}$ before surgery to $5.1^{\circ} \pm 2.4^{\circ}$ at the last follow-up. The patellar tilt angle had significant improvement from $24.5^{\circ} \pm 5.2^{\circ}$ before surgery to $12.30^{\circ} \pm 1.90^{\circ}$ at the last follow-up. The Kujala score was significantly increased from $52.9 \pm 3.2$ points preoperatively to $90.1 \pm 5.8$ points postoperatively $(P<0.05)$. The mean Lysholm score was significantly increased from $47.2 \pm 5.2$ to $92.5 \pm 6.2$ points postoperatively $(P<0.05)$. The Tegner activity score improved overall from $3.1 \pm 0.6$ points to $5.8 \pm 0.9$ points at follow-up.

Conclusion: We have done a simple technique where the MPFL is reconstructed safely to avoid patella fracture, anatomically to restore physiological kinematics and stability, and economically to reduce costs with bone-fascia tunnel fixation at the medial margin of the patella.

Keywords: Patella, Instability, Medial patellofemoral ligament, Reconstruction, Fixation

\footnotetext{
* Correspondence: docshenyong@163.com

'Equal contributors

'Department of Orthopedic Surgery, The Key Laboratory of Orthopedic

Biomechanics of Hebei Province, Third Hospital of Hebei Medical University,

139 Ziqiang Road, Shijiazhuang 050051, China

Full list of author information is available at the end of the article
}

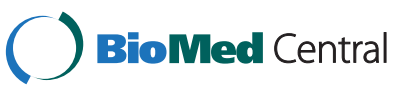

(c) 2014 Li et al.; licensee BioMed Central Ltd. This is an Open Access article distributed under the terms of the Creative Commons Attribution License (http://creativecommons.org/licenses/by/2.0), which permits unrestricted use, distribution, and reproduction in any medium, provided the original work is properly credited. The Creative Commons Public Domain Dedication waiver (http://creativecommons.org/publicdomain/zero/1.0/) applies to the data made available in this article, unless otherwise stated. 


\section{Introduction}

Recurrent lateral dislocation of the patella is difficult to treat because of many complex contributing factors such as generalized ligamentous laxity, inadequate medial retinacular tissue, insufficient trochlear groove restraint, patellar tendon length abnormalities, limb alignment torsional abnormalities, and others [1]. Over 130 different surgical methods have been described which can be classified as proximal realignment, distal realignment, proximal and distal realignment, lateral retinacular release, and medial retinacular placation [2].

The medial patellofemoral ligament (MPFL) was first described by Kaplan [3]. Since Warren and Marshall [4] introduced the concept of a three-layered pattern of the medial capsular ligament of the knee and described the fibers of the MPFL existing within layer II, and biomechanical studies $[5,6]$ had demonstrated that the MPFL accounts for approximately $50 \%$ to $60 \%$ of the total lateral restraint; thus being the primary medial stabilizer of the patella, MPFL reconstruction has became an accepted technique to treat patellofemoral instability. Recently, numerous techniques for reconstruction of the medial patellofemoral complex have been described with promising clinical results [7]. Many authors have described the method of making the patellar tunnel and using the wire anchors to fix the side of the patella. However, drilling tunnels transversely across the patella creates a stress riser and can lead to fracture. The use of wire anchors increase the economic burden of patients and also the patellar bone may be too soft for a secure anchoring. Here, we present a report on MPFL reconstruction for recurrent patellar dislocation with bone-fascia tunnel fixation at the medial margin of the patella. In this article, we discuss a surgical technique for MPFL reconstruction along with some discussion of the anatomic and clinical rationale.

The aim of the study is to evaluate the results of MPFL reconstruction with bone-fascia tunnel fixation at the medial margin of the patella after a 6-year-minimum follow-up. We hypothesized that this procedure is equal with other techniques for MPFL reconstruction, improving patellofemoral joint function and quality of life and giving pain relief.

\section{Methods}

From June 2005 to May 2006, all patients (72 patients) with lateral patellofemoral joint instability had been treated at the Third Hospital of Hebei Medical University. Five patients preferred to conventional treatment, where two of them had combined multiple-ligament injuries. Sixty-five patients who underwent a primary MPFL reconstruction were included in the study. This study was approved by the ethics committee of the Third Hospital of Hebei Medical University. Signed informed consent was obtained from each patient.

Inclusion criteria included patients with a diagnosis of recurrent patellar dislocation, confirmed with history and physical and radiographic examinations. Exclusion criteria for this operation were a history of previous knee surgery; multiple ligament injury; significant patellofemoral articular cartilage degenerative changes according to the Outerbridge classification (grades III-IV); significant patellar malalignment, wherein the tibial tubercle-totrochlear groove distance (TT-TG) is greater than $20 \mathrm{~mm}$; and severe trochlear dysplasia (Table 1).

\section{Evaluation and rating scales}

Demographic data, physical examination, Kujala scale, Lysholm score, and Tegner activity score were completed preoperatively and at each follow-up evaluation. Clinical data were prospectively collected preoperatively and at 3 , $6,12,24,36,48,60$, and 72 months after surgery. When the follow-up was longer than 6 years, the last data available were used for statistical analysis.

\section{Surgical technique}

Examination under anesthesia is performed on both knees to assess for increased lateral translation of the patellar and for a tight lateral retinaculum. A lateral release was performed if the patella was unable to be everted to neutral.

A tourniquet is applied to the thigh and then diagnostic arthroscopy is performed before reconstruction of the MPFL. The cartilaginous situation, tracking of the patella through a range of flexion, and trochlear shape are assessed.

A longitudinal incision $(2-3 \mathrm{~cm})$ is performed on the anteromedial side of the patella, then the soft tissue is separated to the bone surface and the periosteum is peeled back. A bone groove which extends approximately from the superomedial corner of the patella to the midpoint of the medial margin of the patella is created using the rongeur (Figure 1). The groove must be deep enough, about 3 $\mathrm{mm}$, so that the allograft can be completely embedded. Deep-frozen human tibialis anterior allografts were used in all patients. The two ends of the graft are fixed with a non-absorbable braided suture. Then, the diameter of the

Table 1 Demographics of the patients

\begin{tabular}{ll}
\hline Indices & Values \\
\hline Gender (male/female) & $28 / 37$ \\
Knee (left/right) & $33 / 32$ \\
Age at surgery (year) & $29.4 \pm 5.6$ \\
Follow-up time (months) & $78.5 \pm 3.8$ \\
$Q$ angle $\left(^{\circ}\right)$ & $13.4 \pm 2.7$ \\
TT-TG distance (mm) & $14.6 \pm 1.9$ \\
\hline
\end{tabular}




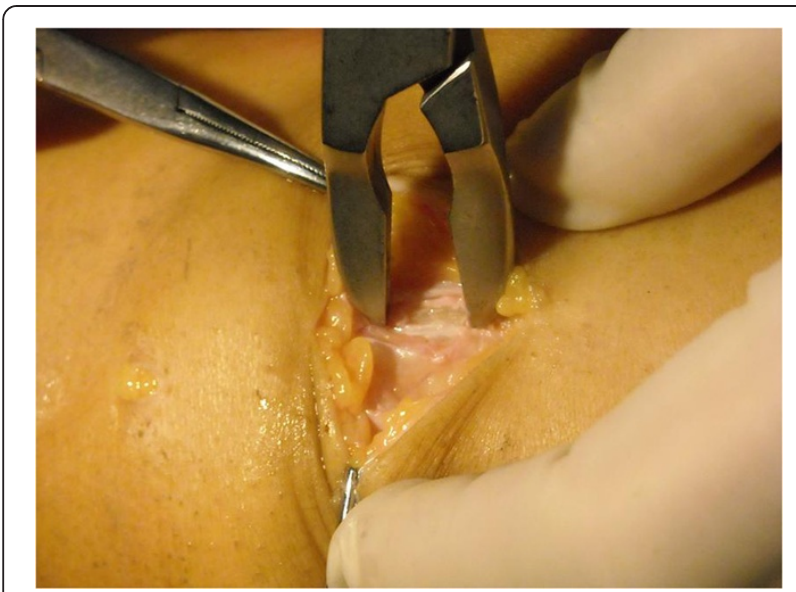

Figure 1 The bone groove is created using the rongeur.

folded graft is measured with a sizing guide. The middle of the allograft is put into the groove, with the patellar fascia overlying. The absorbable sutures are used to fix the graft, so the bone-fascia tunnel is performed (Figure 2). The method of suture is mainly divided into three needles (Figure 3).

A $2-\mathrm{cm}$ incision is made over the femoral attachment of the MPFL which is just distal to the adductor tubercle and superoposterior to the medial femoral epicondyle. Then, the second and the third layers of the medial patellofemoral complex, where the MPFL is anatomically situated [4], are separated from each other down to the femoral insertion side. The free ends of the graft are then pulled through with the help of a vascular clamp.

A guide pin with an eyelet is placed at the anatomical femoral insertion and directed slightly anterior and superior to avoid penetration of the condyle posteriorly. The guide pin is then overdrilled with an appropriately sized cannulated acorn reamer based on sizing of the doubled graft to a depth adequate to allow full seating of the free ends of the graft within the femur. The two graft strands are whipstitched with a No. 2 non-absorbable suture. The sutures are then placed through the eyelet of the guide pin which is then advanced out the contralateral cortex of the distal femur. Next, the grafts are pulled into the femoral tunnel, and they are free and not bottomed out. The knee is cycled for several times from full flexion to full extension with the graft under tension.

Insufficient tension will result in a lack of correction of the lateral instability, whereas excessive tension will cause increased pressure in the patellofemoral joint and may restrict knee range of motion. Therefore, it is essential to find a tolerated tension; the surgeon can arthroscopically adjust the tension and see normal patellar tracking restored during the whole range of knee motion. Once sufficient tension has been obtained, the femoral attachment of the graft is fixed with a bioabsorbable interference screw of the same size as the drill which is used in $30^{\circ}$ of knee flexion. This is because biomechanical studies $[8,9]$ have shown that the MPFL has its maximal restraint against patella lateralization in $30^{\circ}$ of knee flexion.

The whip sutures from the graft ends are then cut under the skin. The wound is closed in layers, and routine dressings, bandages, and a knee brace are applied.

\section{Postoperative rehabilitation}

At the beginning, all patients followed the same rehabilitation protocol after MPFL reconstruction. Postoperatively, the patients were partially weight bearing in full extension with a knee brace. Two weeks after surgery, the patients were allowed to progress from partial to full weight bearing. In 65 patients, 6 patients $(9.2 \%)$ were athletes who returned to sporting activity routine such as weight bearing with the contralateral limb and cycling at 4 weeks after surgery. Then, they started running, and sport-specific rehabilitation programs at 12 weeks after surgery. They return to high-risk sports such as soccer or basketball at 6 months after surgery. Fifty-nine patients (90.8\%) were non-athletes who were allowed to remove the knee brace and began to do aggressive quadriceps, hamstring, and hip
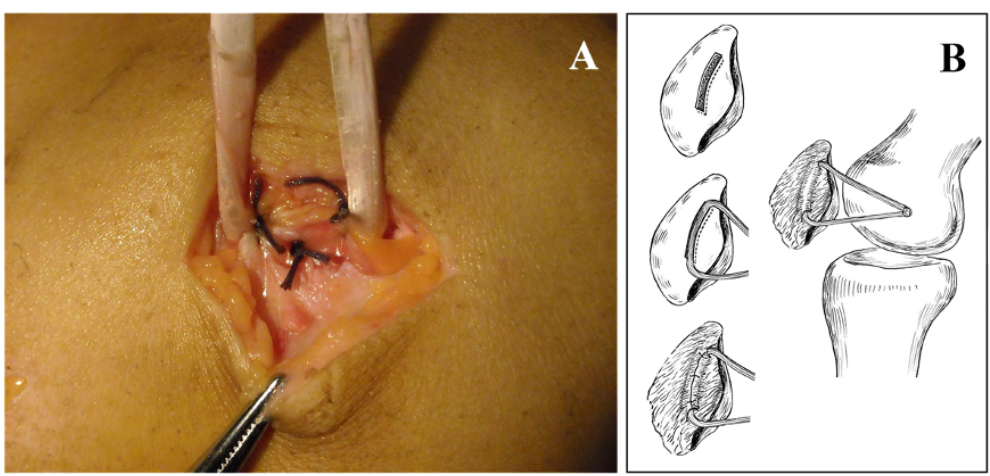

Figure 2 Bone-fascia tunnel is performed (A) and reconstruction of MPFL with proximal and distal bundle (B). 

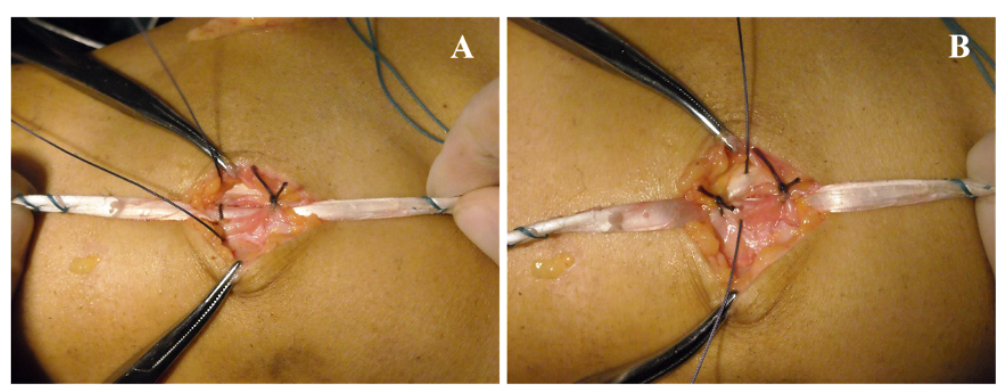

Figure 3 The middle suture needle passes through the tendon graft to limit movement (A, B).

muscle strengthening exercises after 4 weeks. Patients typically returned to normal daily activities at 4 months postoperatively. The time patients returned to daily activity routine or sports was based on their individual level of performance. Furthermore, the function of athletes was acceptable after 6 years.

\section{Statistical analysis}

Statistical analysis was performed with SPSS software (version 11.0; SPSS, Chicago, IL, USA). Preoperative and postoperative indices for this study were compared by paired $t$ test. $P<0.05$ was defined as a significant difference.

\section{Results}

At the last follow-up, all the patients demonstrated a significant improvement compared to the preoperative status. There were no cases of major neurological, vascular, or wound complications. No patient needed additional surgery due to recurrent or residual symptoms. The CT measurement results have significant improvement compared with the preoperative results. The congruence angle had significant improvement from $19.2^{\circ} \pm$ $6.3^{\circ}$ before surgery to $-6.0^{\circ} \pm 0.5^{\circ}$ at the last follow-up. The lateral patellar angle had significant improvement from $-6.9^{\circ} \pm 3.5^{\circ}$ before surgery to $5.1^{\circ} \pm 2.4^{\circ}$ at the last follow-up. The patellar tilt angle had significant improvement from $24.5^{\circ} \pm 5.2^{\circ}$ before surgery to $12.3^{\circ} \pm 1.9^{\circ}$ at the last follow-up (Table 2).

The Kujala score was significantly increased from $52.9 \pm$ 3.2 points preoperatively to $90.1 \pm 5.8$ points postoperatively $(P<0.05)$. The mean Lysholm score was significantly increased from $47.2 \pm 5.2$ to $92.5 \pm 6.2$ points postoperatively $(P<0.05)$. The Tegner activity score improved overall

Table 2 Patellofemoral measurements on computed tomography

\begin{tabular}{llll}
\hline Angle $\left(^{\circ}\right)$ & Preoperation & Postoperation & $\boldsymbol{P}$ value \\
\hline Congruence angle & $19.2 \pm 6.3$ & $-6.0 \pm 0.5$ & 0.000 \\
Lateral patellar angle & $-6.9 \pm 3.5$ & $5.1 \pm 2.4$ & 0.000 \\
Patellar tilt angle & $24.5 \pm 5.2$ & $12.3 \pm 1.9$ & 0.000 \\
\hline
\end{tabular}

from $3.1 \pm 0.6$ points to $5.8 \pm 0.9$ points at follow-up (Table 3).

\section{Discussion}

MPFL reconstruction has become an accepted technique to treat patellofemoral instability. Numerous surgical techniques have been described to reconstruct the MPFL. The graft of the femoral side is almost secured in the femoral tunnel with an absorbable interference screw, but for the fixation of the patellar side, it includes a few techniques for the reconstruction of this ligament, making the patellar tunnel; and using the wire anchors is described by many authors. The direction of the bone tunnel varied from parallel to oblique. In 2003, Nomura and Inoue [10] described the patellar tunnel which was drilled from the proximal third of the medial margin of the patella to the center of the anterior aspect of the patella. In 2007, Carmont and Maffulli [11] described a similar technique of drilling the bone tunnels that traverse the entire patella; and in 2009, Papp and Cosgarea [12] described the blind patellar tunnel which was drilled from medial to lateral at the midpoint of the MPFL insertion.

Gomes [13] warned against damaging the cartilage surface of the patella by creating a bone tunnel. Dobbs et al. [14] was able to show that drilling tunnels transversely across the patella creates a stress riser and can lead to fracture. This complication has been seen using a previously described technique [1] where Fithian and Gupta [15] reported a 305-lb 17-year-old male who fell 1 year after reconstruction of MPFL by drilling two tunnels on the patella which enter at the medial articular margin and exit at the anterior (ventral) patellar surface. $\mathrm{X}$-rays showed that the medical bone bridge had fractured. Mikashima et al. [16] reported that no significant

Table 3 Analysis of outcomes according to functional score

\begin{tabular}{llll}
\hline & Preoperation & Postoperation & $\boldsymbol{P}$ value \\
\hline Kujala score & $52.9 \pm 3.2$ & $90.1 \pm 5.8$ & 0.000 \\
Lysholm score & $47.2 \pm 5.2$ & $92.5 \pm 6.2$ & 0.000 \\
Tegner activity score & $3.1 \pm 0.6$ & $5.8 \pm 0.9$ & 0.000 \\
\hline
\end{tabular}


difference after a 2-year follow-up was observed in the quality of fixation between suturing to the periosteum and the fibrous tissue overlying the patella and fixation in a bone tunnel. They had two patella fractures whose reconstructed MPFL was fixed into a bone tunnel in the patella.

In 2008, Schottle et al. [7] described their technique of securing the graft to the medial patella with two suture anchors and to the femur with a biodegradable interference screw. The pull of the reconstructed MPFL on the patellar side is over the femoral drill tunnel and extended to the proximal suture anchor, where it is fixed by knots. The fixation strength is mainly between the tendon and suture. As a result of the suture anchor, the tension on the tendon-to-bone contact increased which did not help tendon-to-bone healing, as compared with the pressure contact. The use of the suture anchor also increased the economic burden of patients and also the patellar bone may be too soft for a secure anchoring.

We make the bone-fascia tunnel at the medial margin of the patella. The cancellous bone of the bone groove makes up $2 / 3$ of the tunnel, and the remaining $1 / 3$ is formed by the covered fascia. In addition to the medially directed force to the patella, the reconstructed MPFL also provides a posterior force; therefore, it is a posteromedial force on the patella. Such a force may limit the graft in the bone groove to be well associated with the firmly sutured fascia. So, the fixation of the graft at the patellar side by the bone-fascia tunnel technique permits secure fixation and direct pull on the patella, which has mechanical characteristics similar to those of the bone tunnel technique. The tendon-to-bone contact is pressure contact. The graft is also permitted to heal in a bone tunnel, allowing for increased surface area for graft-to-bone healing. This eliminates the risks of patellar fracture and violation of the patellar articular surface.

In 2004, Steensen et al. [17] described that the average width of the patellar insertion was larger than that of the femoral insertion. In 2008, Parker et al. [18] evaluated and compared the patellofemoral kinematics of a single-stranded isometric medial patellofemoral ligament reconstruction technique with that of a doublestranded anatomic technique that more closely recreates the normal anatomy of the medial patellofemoral ligament.

In our technique for MPFL reconstruction, the making of the bone groove at the patellar side and the use of allograft allows us to place the graft at the anatomical insertion and recreate the double-bundle structure of the MPFL and a sail-like triangular shape of the graft, which is comparable to the original anatomy $[8,19]$. Reconstruction of the MPFL using the double-bundle anatomic technique creates a proximal and a distal bundle [8] and reproduces the anatomy of the native MPFL. This seems to provide a higher stability during flexion and decreases the patella rotation in contrast to a singlebundle isometric technique.

\section{Limitations}

There were several limitations to our study, including the non-randomized and retrospective study design. The greatest limitations were the small number of patients and the lack of a control group. In the future study, we will enroll more patients and compare different MPFL reconstruction methods to observe which method is associated with lower morbidity and more rapid rehabilitation, which might be beneficial for such patients.

\section{Conclusion}

This method for MPFL reconstruction is a relatively easy and safe procedure that provides enough graft strength comparable to previous techniques. This is a simple technique where the MPFL is reconstructed safely to avoid patella fracture, anatomically to restore physiological kinematics and stability, and economically to reduce costs with bone-fascia tunnel fixation at the medial margin of the patella. Further studies are in progress.

\section{Competing interests}

The authors declare that they have no competing interests.

\section{Authors' contributions}

$J L$ and $Y L$ carried out the conception and design of the study, acquisition and interpretation of data, and drafted the manuscript. JW drafted the manuscript substantially. JW and SG carried out the acquisition and interpretation of data. YS revised the manuscript critically for important intellectual content and gave final approval of the version to be published. All authors read and approved the final manuscript.

\section{Acknowledgements}

We thank Dr. Yingze Zhang for his support to obtain the approval of the ethics committee for this study.

\section{Author details}

'Department of Orthopedic Surgery, The Key Laboratory of Orthopedic Biomechanics of Hebei Province, Third Hospital of Hebei Medical University, 139 Ziqiang Road, Shijiazhuang 050051, China. ${ }^{2}$ Department of Orthopedic Surgery, Hebei General Hospital, 348 Heping Road, Shijiazhuang 050000, China.

Received: 26 January 2014 Accepted: 15 July 2014

Published: 15 August 2014

\section{References}

1. Schock EJ, Burks RT: Medial patellofemoral ligament reconstruction using a hamstring graft. Oper Tech Sports Med 2001, 9:169-175.

2. Insall JN: Disorders of the patella. In Surgery of the Knee. Edited by Insall JN. New York: Churchill Livingstone; 1984:191-260.

3. Kaplan EB: Factors responsible for the stability of the knee joint. Bull Hosp Joint Dis 1957, 18:51-59.

4. Warren LF, Marshall $\mathrm{J}$ : The supporting structures and layers on the medial side of the knee: an anatomical study. J Bone Joint Surg Am 1979, 61:56-62.

5. Conlan T, Garth WP Jr, Lemons JE: Evaluation of the medial soft-tissue restraints of the extensor mechanism of the knee. J Bone Joint Surg 1993, 75:682-693.

6. Desio SM, Burks RT, Bachus KN: Soft tissue restraints to lateral patellar translation in the human knee. Am J Sports Med 1998, 26:59-65. 
7. Schottle PB, Romero J, Schmeling A, Weiler A: Technical note: anatomical reconstruction of the medial patellofemoral ligament using a free gracilis autograft. Arch Orthop Trauma Surg 2008, 128:479-484.

8. Amis AA, Firer P, Mountney J, Senavongse W, Thomas NP: Anatomy and biomechanics of the medial patellofemoral ligament. Knee 2003, 10:215-220.

9. Davis DK, Fithian DC: Techniques of medial retinacular repair and reconstruction. Clin Orthop Relat Res 2002, 402:38-52.

10. Nomura $\mathrm{E}$, Inoue M: Technical note: surgical technique and rationale for medial patellofemoral ligament reconstruction for recurrent patellar dislocation. Arthroscopy 2003, 19:E47.

11. Carmont MR, Maffulli N: Medial patellofemoral ligament reconstruction: a new technique. BMC Musculoskelet Disord 2007, 8:22.

12. Papp DF, Cosgarea AJ: Medial patellofemoral ligament reconstruction for recurrent patellar instability. Tech Knee Surg 2009, 8:187-193.

13. Gomes JL: Medial patellofemoral ligament reconstruction for recurrent dislocation of the patella: a preliminary report. Arthroscopy 1992 8:335-340.

14. Dobbs RE, Greis PE, Burks RT: Medial patellofemoral ligament reconstruction. Tech Knee Surg 2007, 6:29-36.

15. Fithian DC, Gupta N: Patellar instability: principles of soft tissue repair and reconstruction. Tech Knee Surg 2006, 5:19-26.

16. Mikashima Y, Kimura M, Kobayashi Y, Miyawaki M, Tomatsu T: Clinical results of isolated reconstruction of the medial patellofemoral ligament for recurrent dislocation and subluxation of the patella. Acta Orthop Belg 2006, 72:65-71.

17. Steensen RN, Dopirak RM, McDonald WG III: The anatomy and isometry of the medial patellofemoral ligament: implications for reconstruction. Am J Sports Med 2004, 32:1509-1513.

18. Parker DA, Alexander JW, Conditt MA, Uzodinma ON, Bryan WJ: Comparison of isometric and anatomic reconstruction of the medial patellofemoral ligament. Orthopedics 2008, 31:339-343.

19. Nomura E, Inoue M, Osada N: Anatomical analysis of the medial patellofemoral ligament of the knee, especially the femoral attachment. Knee Surg Sports Traumatol Arthrosc 2005, 13:510-515.

doi:10.1186/s13018-014-0066-7

Cite this article as: Li et al.: A simple technique for reconstruction of medial patellofemoral ligament with bone-fascia tunnel fixation at the medial margin of the patella: a 6-year-minimum follow-up study. Journal of Orthopaedic Surgery and Research 2014 9:66.

\section{Submit your next manuscript to BioMed Central and take full advantage of:}

- Convenient online submission

- Thorough peer review

- No space constraints or color figure charges

- Immediate publication on acceptance

- Inclusion in PubMed, CAS, Scopus and Google Scholar

- Research which is freely available for redistribution

Submit your manuscript at www.biomedcentral.com/submit
(O) Biomed Central 